\title{
A General Synthesis of Benzoazepinoindoles - A New Class of Heterocycles
}

\author{
Jeremy C. Dobrowolskia \\ Duyen H.T. Nguyen ${ }^{a}$ \\ Benjamin H. Fraser ${ }^{\text {b }}$ \\ Mohan Bhadbhade ${ }^{c}$ \\ David StC. Black ${ }^{a}$ \\ Naresh Kumar*a \\ a School of Chemistry, The University of New South Wales, Sydney, NSW \\ 2052, Australia \\ n.kumar@unsw.edu.au \\ b The Australian Nuclear Science and Technology Organisation, Kirrawee DC, \\ NSW 2232, Australia \\ ' Solid State \& Elemental Analysis Unit, Mark Wainwright Analytical Centre, \\ Division of Research, The University of New South Wales, Sydney, NSW \\ 2052, Australia
}

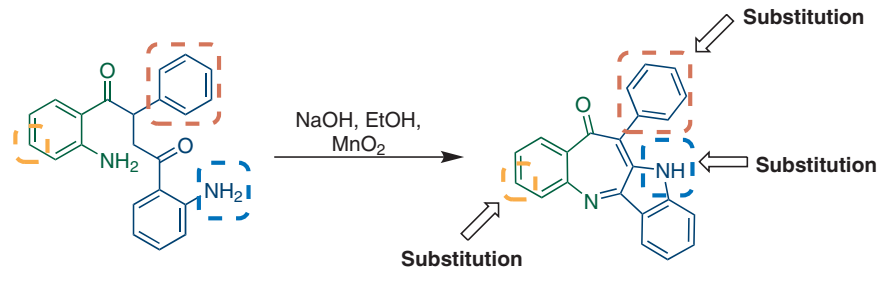

New class of heterocycles

- Highly functionalisable structures $\quad 43-68 \%$ Yield

- Efficient two-step synthesis

- Inexpensive
Received: 14.08.2019

Accepted after revision: 18.09.2019

Published online: 09.10 .2019

DOI: 10.1055/s-0039-1690700; Art ID: st-2019-d0360-I

Abstract A new class of heterocyclic compounds, namely the benzoazepinoindolones, has been synthesised through a base-catalysed cyclisation reaction of 1,4-bis(2-aminophenyl)-2-phenylbutane-1,4-dione derivatives and features the prominent seven-membered azepine ring moiety. This synthesis has considerable scope for the rapid generation of more complex structures and is inexpensive and generally applicable.

Key words dihydrobenzo[6,7]azepino[3,2-c]indolones, fused-ring systems, umpolung, condensation, heterocycles

Heterocyclic compounds, in particular those containing the indole moiety, are a core focus for medicinal chemists due to the inherent range of biological properties associated with them. The indole moiety can be found in many natural products and has the potential for the introduction of high degrees of functionality. ${ }^{1}$ Various indole-containing compounds have found applications in the treatment of cancer, ${ }^{2}$ bacterial infections, ${ }^{2-4}$ and type-II diabetes. ${ }^{5}$

Similarly, compounds containing the benzazepine scaffold have attracted significant attention due to their broad applicability, most prominently as drugs for the treatment of heart conditions ${ }^{6}$ and neuropsychiatric disorders ${ }^{7}$ as well as in the treatment of various cancers. ${ }^{8}$

Fusion of the indole moiety with the benzazepine scaffold would generate an interesting collection of compounds with potentially diverse biological properties. Although various approaches have been used to generate indole and benzazepine derivatives, a suitable synthetic route to fused indole and benzazepine derivatives is required. To date, there are no reports of the synthesis of dihydroben- zo[6,7]azepino[3,2-c]indoles and only limited reports relating to the synthesis and functionalisation of the benzoazepine scaffold with other heterocycles., ${ }^{9,10}$

Previously, we described the generation of 1,4-bis(2aminophenyl)-2-phenylbutane-1,4-diones through a basecatalysed addition, oxidation, and ring-cleavage process starting with 2-aminochalcones and 2-oxindole. We showed that the second amine functionality is crucial for the generation of the novel benzo[6,7]azepino[3,2-c]quinolinones. ${ }^{9}$

In this work we have developed a two-step synthesis of a new class of heterocycles, namely the benzo[6,7]azepino[3,2-c]indolones. The initial base-catalysed reaction of 2aminochalcone with 2-oxindole using manganese dioxide and DMSO gives the intermediate 1,4-bis(2-aminophenyl)2-phenylbutane-1,4-dione, and a subsequent base-catalysed reaction with manganese dioxide and ethanol gives the previously unreported benzo[6,7]azepino[3,2-b]indol$12(10 H)$-one ring system (Scheme 1 ).<smiles>Nc1ccccc1C(=O)/C=C/c1ccccc1</smiles>
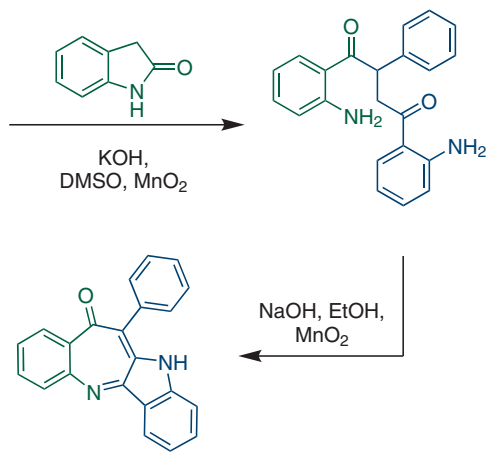

Scheme 1 Accessing a new class of heterocycles: benzo[6,7]azepino[3,2-c]indolones 
The formation of the novel benzo[6,7]azepino[3,2-c]indolones is possible through an oxidative cyclisation of diamino-1,4-diketones, which have been previously reported by our group. ${ }^{9}$ However, these are now more readily available through an improved synthesis using potassium hydroxide and manganese dioxide in DMSO. In order to optimise the conditions for the condensation reaction, 1,4bis(2-aminophenyl)-2-(4-iodophenyl)butane-1,4-dione (1) was used as a model substrate to generate 11-(4-iodophenyl)benzo[6,7]azepino[3,2-b]indol-12(10H)-one (2, Scheme 2, Table 1).

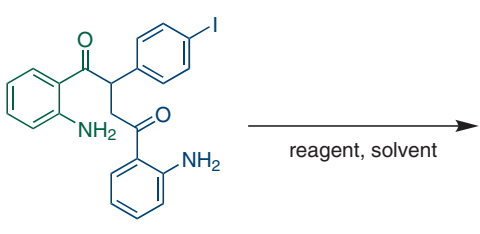

1

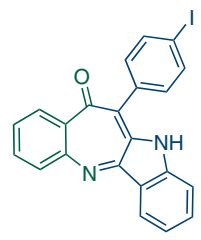

2
Scheme 2 Conversion of 1,4-bis(2-aminophenyl)-2-(4-iodophenyl)butane-1,4-dione 1 into 11-(4-iodophenyl)benzo[6,7]azepino[3,2-b]indol12(10H)-one (2)

Table 1 Optimisation of Condensation Reaction to Generate 11-(4-Iodophenyl)benzo[6,7]azepino[3,2-b]indol-12(10H)-one (2)

\begin{tabular}{lllccc}
\hline Entry & Reagent & Solvent & \multicolumn{2}{c}{ Time $(\mathrm{h})$} & Temp $\left({ }^{\circ} \mathrm{C}\right)$ \\
\hline 1 & PTSA & EtOH & 24 & 80 & 0 \\
2 & PTSA & $2-\mathrm{PrOH}$ & 24 & 80 & 0 \\
3 & $\mathrm{KOH}$ & DMSO & 48 & 80 & 0 \\
4 & $\mathrm{NaOH}$ & DMSO & 48 & 80 & 0 \\
5 & $\mathrm{NaOH}$ & EtOH & 48 & 80 & 49 \\
6 & $\mathrm{NaOH}$ & EtO Hair bubbled & 48 & 80 & 49 \\
7 & $\mathrm{NaOH} / \mathrm{MnO}_{2}$ & EtOH & 48 & 80 & 60 \\
\hline
\end{tabular}

Under acidic conditions several byproducts were generated, none of which were the desired compound (Table 1, entries 1 and 2). Strongly basic conditions in DMSO did not give the desired product (Table 1, entries 3 and 4); whereas use of sodium hydroxide in ethanol, either with or without bubbled air, gave a moderate yield of the desired product (Table 1, entries 5 and 6 ). The addition of manganese dioxide (1 equiv) improved the yield of 11-(4-iodophenyl)ben-
zo[6,7]azepino[3,2-b]indol-12(10H)-one (2) to 60\% (Table 1, entry 7). Increasing the amount of manganese dioxide gave no further improvement.

The benzo[6,7]azepino[3,2-c]indolone moiety presumably arises through the initial imine formation between the primary amine featured in the (2-aminophenyl)(oxo)methanide and the 4-carbonyl group of the 1,4-bis(2-aminophenyl)-2-(4-iodophenyl)butane-1,4-dione (1) to give 2-(2aminophenyl)-4-(4-iodophenyl)-3,4-dihydro-5H-benzo[b]azepin-5-one (3). An oxidation step generates the $\alpha, \beta$ unsaturated ketone $\mathbf{4}$ allowing for nucleophilic attack of the remaining free amine to form the 11-(4-iodophenyl)10,10a-dihydrobenzo[6,7]azepino[3,2-b]indol-12-ol (5), with a final oxidation to give the desired 11-(4-iodophenyl)benzo[6,7]azepino[3,2-b]indol-12(10H)-one (2, Scheme 3).<smiles>Cc1ccc(C(CC(=O)c2ccccc2N)C(=O)c2ccccc2N)cc1</smiles>

1

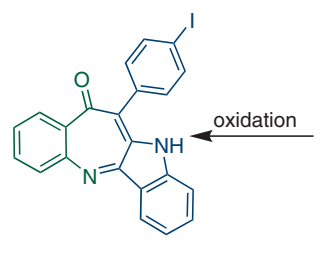

2
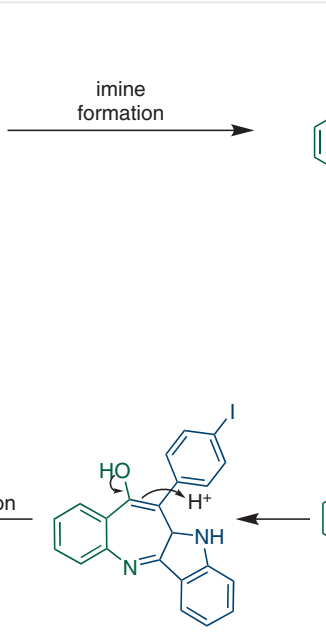
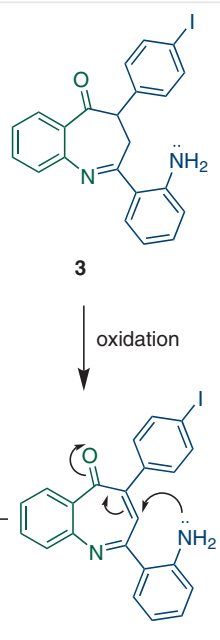

Scheme 3 Proposed mechanism for formation of $\mathbf{2}$

Alternatively, oxidation of $\mathbf{1}$ to an ene-dione structure $\mathbf{6}$ would allow for the formation of a 3 -indolinone 7 , which could undergo imine formation and subsequent oxidation to give the observed product $\mathbf{2}$ (Scheme 4 ).

The reaction is general and tolerates both electronwithdrawing and electron-donating groups on the pendant phenyl ring. The structures of compounds $\mathbf{9 a - 0}$ (Table 2) were determined by spectroscopic analysis. For example, the ${ }^{1} \mathrm{H}$ NMR spectrum of compound $9 \mathbf{a}$ showed a signal at $\delta=10.10 \mathrm{ppm}$ corresponding to the indole $\mathrm{NH}$. The struc-
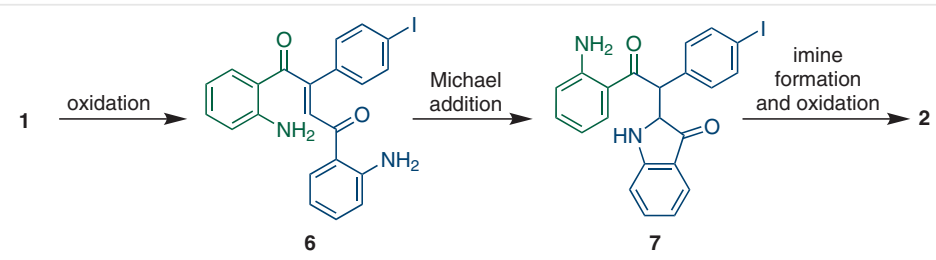

Scheme 4 Alternative mechanism for formation of compound $\mathbf{2}$ 
ture of 9a matched the expected molecular mass of $m / z=$ 357.0788 and was further confirmed by X-ray crystallographic analysis (Figure 1).

Table 2 Synthesised Benzo[6,7]azepino[3,2-b]indolones 9
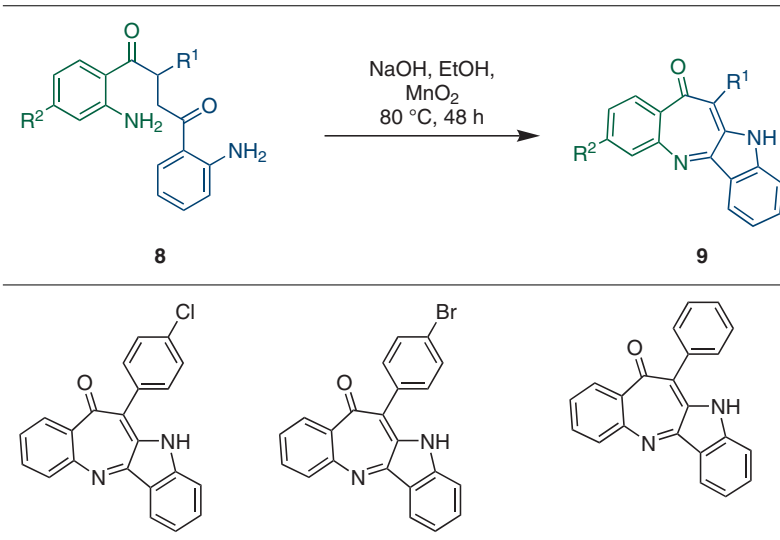

9a $56 \%$

9b $50 \%$

$9 c 47 \%$
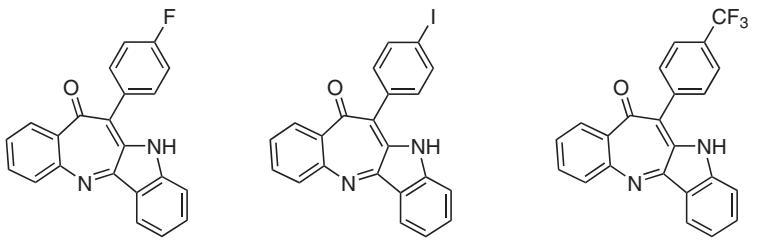

9d $68 \%$

9e $60 \%$

9f $67 \%$


9g $43 \%$<smiles>O=c1c(-c2cccs2)c2[nH]c3ccccc3nc-2c2ccccc12</smiles>

9h $56 \%$

9i $52 \%$<smiles></smiles>

9j $64 \%$<smiles></smiles>

$9 \mathbf{k} 53 \%$<smiles></smiles>

$9 m 50 \%$

9n $62 \%$<smiles></smiles>

9l $65 \%$

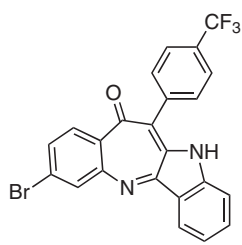

9o $51 \%$

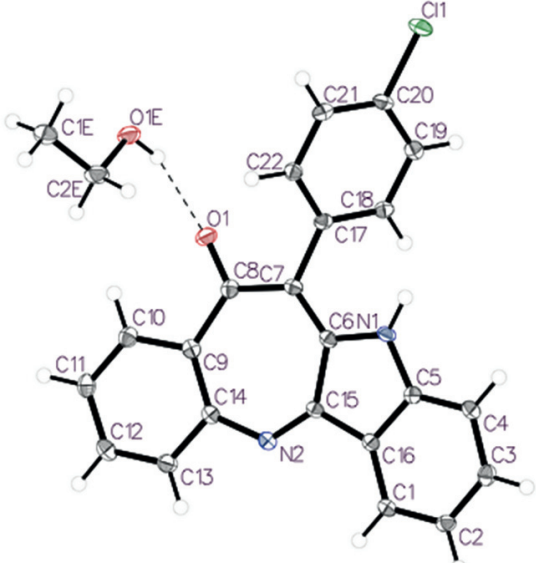

Figure 1 ORTEP diagram of compound 9a

It would be logical to assume that a direct one-pot synthesis of dihydrobenzo[6,7]azepino[3,2-c]indolones is possible, starting with 2 -aminochalcones and oxindoles. To explore this possibility, we reacted (E)-1-(2-aminophenyl)-3(4-chlorophenyl)prop-2-en-1-one (10) with 2-oxindole (11) under a range of conditions (Scheme 5).

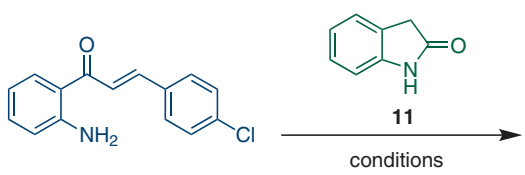

10

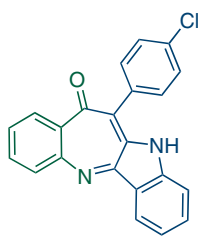

9a
Scheme 5 Attempted one-pot synthesis of $\mathbf{9 a}$

The conditions included a variety of organic and inorganic bases as well as solvents and temperatures, but the desired 11-(4-chlorophenyl)benzo[6,7]azepino[3,2-b]indol-12(10H)-one (9a) could only be generated in very limited yields. At best, only $11 \%$ of the desired final product was obtained when potassium carbonate and DMSO were used at $80{ }^{\circ} \mathrm{C}$. Increasing the temperature further to $150{ }^{\circ} \mathrm{C}$ resulted in a large amount of byproducts, whereas conducting the reaction at room temperature gave only traces of product. Use of other solvents such as DMF or acetonitrile provided no improvement. Changing to a stronger base such as sodium hydroxide or potassium hydroxide resulted in no product formation and use of a range of organic or inorganic bases led to complex reaction mixtures and only trace amounts of product.

Improvements were made to the previously reported 1,4-diketone synthesis, ${ }^{9}$ with the use of potassium hydroxide and manganese dioxide in DMSO significantly improving the yield and ease of purification of the 1,4-bis(2-aminophenyl)-2-phenylbutane-1,4-diones. Additionally, previ- 
ously unreported 1,4-bis(2-aminophenyl)-2-phenylbutane1,4-dione derivatives were successfully synthesised using the new method.

Given the improved method for the synthesis of the 1,4bis(2-aminophenyl)-2-phenylbutane-1,4-diones and the subsequent base-catalysed oxidative cyclisation reaction to generate the desired benzo[6,7]azepino[3,2-b]indol-12ones, the two-step synthesis is clearly preferred.

In an attempt to isolate the intermediate 2-(2-aminophenyl)-4-(4-iodophenyl)-3,4-dihydro-5H-benzo[b]azepin5-one 3, N-protected 1,4-bis(2-aminophenyl)-2-(4-iodophenyl)butane-1,4-diones were used. However, when $N$ \{2-[4-(2-aminophenyl)-3-(4-iodophenyl)-4-oxobutanoyl] phenyl $\}$ acetamide (12) or tert-butyl \{2-[4-(2-aminophenyl)-3-(4-iodophenyl)-4-oxobutanoyl]phenyl\}carbamate (13) were used with sodium hydroxide and manganese dioxide in ethanol, both reactions gave the same isolated product, namely the final 11-(4-iodophenyl)benzo[6,7]azepino[3,2-b]indol-12(10H)-one (2, Scheme 6). This suggests that deprotection of the amide to a free amine takes place before formation of the 5-membered ring.

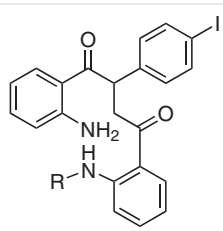

$12 \mathrm{R}=\mathrm{COCH}_{3}$

$13 \mathrm{R}=\mathrm{COOC}\left(\mathrm{CH}_{3}\right)_{3}$
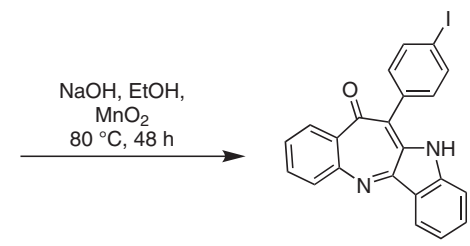

$256-60 \%$
Scheme 6 Deprotection of the amide to generate 11-(4-iodophenyl)benzo[6,7]azepino[3,2-b]indol-12(10H)-one (2)

Alternatively, reaction of 1-[2-(benzylamino)phenyl]-3(4-fluorophenyl)prop-2-en-1-one (14) with oxindole 11 gave the 1-(2-aminophenyl)-4-(2-(benzylamino)phenyl)-2(4-fluorophenyl)butane-1,4-dione (15) and subsequent cyclisation under the previously used conditions gave the $\mathrm{N}$ substituted 10-benzyl-11-(4-fluorophenyl)benzo[6,7]azepino[3,2-b]indol-12(10H)-one (16, Scheme 7).

The structure of compound $\mathbf{1 6}$ was determined by spectroscopic analysis with the ${ }^{1} \mathrm{H}$ NMR spectrum showing a singlet at $\delta=4.64 \mathrm{ppm}$ corresponding to the benzyl group. The structure of compound $\mathbf{1 6}$ matched the expected molecular mass of $m / z=431.1554$ and was further confirmed by X-ray crystallographic analysis (Figure 2 ).

An AlamarBlue assay was used to test the 11-phenylbenzo[6,7]azepino[3,2-b]indol-12(10H)-one derivatives against breast cancer adenocarcinoma cells (MCF-7 cells). The novel analogues display inhibitory activity against the growth of MCF-7 cells. For example, compound 9a was found to have an $\mathrm{IC}_{50}$ of $72.9 \mu \mathrm{M}$ and compound 9 fisplayed similar activity of $73.8 \mu \mathrm{M}$. Therefore, there is po-

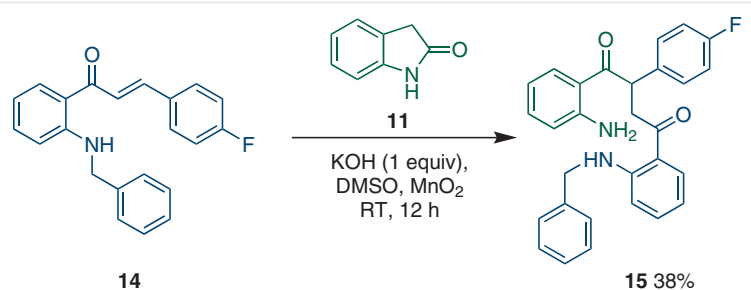

14

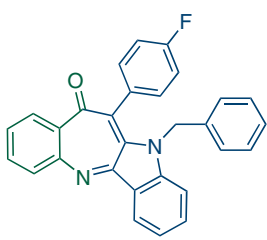

$1538 \%$

$1612 \%$

Scheme 7 Synthesis of 10-benzyl-11-(4-fluorophenyl)benzo[6,7]azepino[3,2-b]indol-12(10H)-one (16)

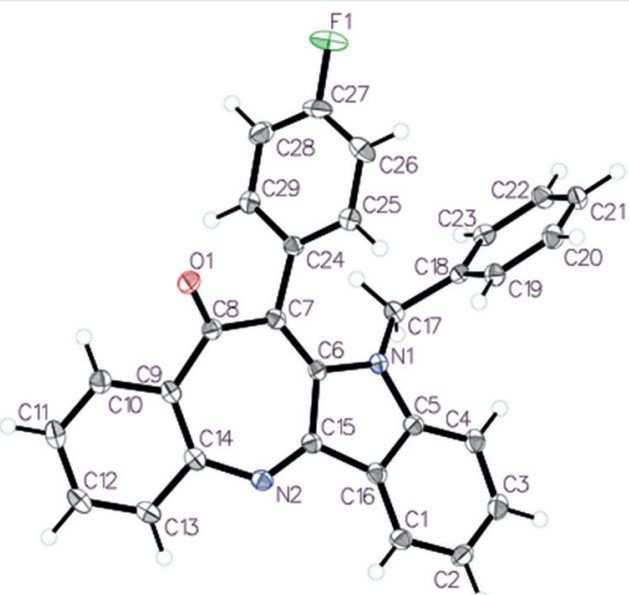

Figure 2 ORTEP diagram of compound 16

tential to explore further and develop biologically active compounds based on this novel scaffold.

In summary, the rapid access to 11-phenylbenzo[6,7]azepino[3,2-b]indol-12(10H)-one derivatives has been achieved through a robust synthetic pathway proceeding via an efficient two-step procedure with initial formation of the 1,4-bis(2-aminophenyl)-2-phenylbutane-1,4-dione intermediates, followed by a base-catalysed oxidative cyclisation reaction. The described synthesis has broad substrate scope and can be used to access a new class of heterocyclic compounds, namely the benzo[6,7]azepino[3,2-b]indol-12ones, in moderate to high yields. This synthetic strategy is efficient and low cost and provides quick access to the desirable azepine moiety that can be seen in a range of drugs on the market today. These novel structures open the possibility of generating new biologically active compounds. 


\section{Funding Information}

J.C.D. thanks University of New South Wales (UNSW) for an Australian Postgraduate Award (APA) and Australian Institute for Nuclear Science and Engineering (AINSE) for a Residential Student Scholarship (RSS). We thank the Australian Research Council (ARC, Grant Number DP180100845) for funding.

\section{Acknowledgment}

We thank the University of New South Wales and the Mark Wainwright Analytical Center for their facilities and support.

\section{Supporting Information}

Supporting information for this article is available online at https://doi.org/10.1055/s-0039-1690700.

\section{References and Notes}

(1) Singh, T. P.; Singh, O. M. Mini-Rev. Med. Chem. 2018, 18, 9.

(2) Lindsay, A. C.; Kim, S. H.; Sperry, J. Nat. Prod. Rep. 2018, 35, 1347.

(3) Melander, R. J.; Minvielle, M. J.; Melander, C. Tetrahedron 2014, $70,6363$.

(4) Mielczarek, M.; Thomas, R. V.; Ma, C.; Kandemir, H.; Yang, X.; Bhadbhade, M.; Black, D. S.; Griffith, R.; Lewis, P. J.; Kumar, N. Bioorg. Med. Chem. 2015, 23, 1763.

(5) Lamotte, Y.; Martres, P.; Faucher, N.; Laroze, A.; Grillot, D.; Ancellin, N.; Saintillan, Y.; Beneton, V.; Gampe, R. T. Bioorg. Med. Chem. Lett. 2010, 20, 1399.

(6) Fukuda, H.; Ito, S.; Watari, K.; Mogi, C.; Arisawa, M.; Okajima, F.; Kurose, H.; Shuto, S. ACS Med. Chem. Lett. 2016, 7, 493.

(7) Tsvelikhovsky, D.; Buchwald, S. L. J. Am. Chem. Soc. 2010, 132, 14048.

(8) Al-Qawasmeh, R. A.; Lee, Y.; Cao, M.-Y.; Gu, X.; Viau, S.; Lightfoot, J.; Wright, J. A.; Young, A. H. Bioorg. Med. Chem. Lett. 2009, 19, 104.

(9) Dobrowolski, J. C.; Nguyen, D. H. T.; Fraser, B. H.; Bhadbhade, M.; Black, D. S.; Kumar, N. Synlett 2019, 30, 567.

(10) Miao, C.-B.; Zeng, Y.-M.; Shi, T.; Liu, R.; Wei, P.-F.; Sun, X.-Q.; Yang, H.-T. J. Org. Chem. 2016, 81, 43. 